REFERENCIA: Martínez-Hita, M. \& Miralles Martínez, P. (2021). Evolución de las concepciones del alumnado de Educación Primaria sobre la Historia como disciplina. ENSAYOS, Revista de la Facultad de Educación de Albacete, 36(1). Enlace web: http://www.revista.uclm.es/index.php/ensayos - Consultada en fecha (dd-mm-aaaa)

\title{
EVOLUCIÓN DE LAS CONCEPCIONES DEL ALUMNADO DE EDUCACIÓN PRIMARIA SOBRE LA HISTORIA COMO DISCIPLINA
}

\section{EVOLUTION OF PRIMARY SCHOOL STUDENTS' CONCEPTIONS OF HISTORY AS A DISCIPLINE}

María Martínez-Hita

m.martinezhita@um.es

Pedro Miralles Martínez

pedromir@um.es

Facultad de Educación. Universidad de Murcia (España)

Recibido: 09/03/2021

Aceptado: 30/06/2021

\section{Resumen:}

El objetivo de esta investigación cuasiexperimental fue analizar la evolución de las concepciones sobre la historia como disciplina del alumnado de 4.ㅇ de Educación Primaria, comparando un grupo experimental que siguió una metodología activa basada en el pensamiento histórico con un grupo control que trabajó con una metodología tradicional. Antes y después de la intervención en el aula, el alumnado respondió a cuatro ítems de una prueba de rendimiento mixta. Los resultados mostraron diferencias estadísticamente significativas en la comparación intergrupal en el postest e intragrupal, especialmente en el grupo experimental. Por ello, se sugiere la implementación de metodologías activas basadas en el pensamiento histórico para mejorar el proceso de enseñanza-aprendizaje de la historia y las concepciones del alumnado sobre la misma.

Palabras clave: concepciones sobre historia; enseñanza de la historia; pensamiento histórico; educación primaria; métodos didácticos.

\footnotetext{
${ }^{1}$ Este trabajo es resultado del proyecto de investigación "El pensamiento geográfico e histórico del alumnado de Educación Primaria en la Región de Murcia: propuesta metodológica innovadora para una educación de calidad" (20874/PI/18). Proyecto financiado por la Comunidad Autónoma de la Región de Murcia a través de la convocatoria de Ayudas a proyectos para el desarrollo de investigación científica y técnica por grupos competitivos, incluida en el Programa Regional de Fomento de la Investigación Científica y Técnica (Plan de Actuación 2018) de la Fundación Séneca-Agencia de Ciencia y Tecnología de la Región de Murcia.
} 


\section{Abstract:}

The aim of this quasi-experimental research was to analyze the evolution of the conceptions of history as a discipline of 4th grade Primary School students, comparing an experimental group that followed an active methodology based on historical thinking with a control group which followed a traditional methodology. Before and after the classroom intervention, the students answered four items that were part of a mixed performance test. The results showed statistically significant differences in the intergroup comparison in the posttest and intragroup comparison, especially in the experimental group. Therefore, we suggest the implementation in the classroom of active methodologies based on historical thinking in order to improve the teachinglearning process of history and the students' conceptions of it.

Keywords: perception of history; history education; historical thinking; primary education; teaching methods.

\section{Introducción}

El pensamiento histórico está adquiriendo progresivamente una mayor popularidad en las investigaciones internacionales sobre la educación histórica (Thorp y Persson, 2020). Eso está teniendo una influencia sobre la enseñanza de la historia en la que, gradualmente, está perdiendo protagonismo el aprendizaje memorístico de los contenidos históricos en favor de un aprendizaje activo de los métodos de investigación histórica, es decir, del desarrollo del pensamiento histórico (Lévesque y Clark, 2018). Además, este enfoque de enseñanza de la historia está en línea con las actuales tendencias educativas que abogan por la incorporación de metodologías activas en el aula (Gómez et al., 2018; Muntaner et al., 2020).

Una enseñanza basada en el pensamiento histórico requiere de un conocimiento sobre cómo se construye el conocimiento histórico, así como sobre el trabajo de los historiadores. La visión que el alumnado tiene sobre la historia como disciplina y la construcción del conocimiento puede favorecer o dificultar la comprensión histórica (Miguel-Revilla et al., 2020; VanSledright y Maggioni, 2016).

Sin embargo, el alumnado suele presentar unas concepciones sobre la historia incompletas o erróneas y un desconocimiento sobre en qué consiste la labor de los historiadores o la utilidad de las fuentes históricas (Brophy y VanSledright, 1992; Brophy et al., 1992; Fraile-Delgado, 2020; Fuentes, 2002, 2006; Miguel-Revilla et al., 2020; Shemilt, 1987a, 1987b).

La metodología utilizada en el aula influye en las concepciones que el alumnado tiene sobre la historia, pudiendo ser modificadas positivamente con una metodología activa y basada en las competencias de pensamiento histórico (Brophy et al., 1992; Fraile-Delgado, 2020; Fuentes, 2002, 2006; Miguel-Revilla et al., 2020; Shemilt, 1987a, 1987b).

No obstante, las investigaciones previas suelen estudiar a discentes de niveles educativos superiores (VanSledright y Maggioni, 2016), existiendo escasos estudios sobre alumnado de Educación Primaria.

En esta investigación pretendemos contribuir a reducir esta laguna en la investigación analizando las concepciones sobre la historia como disciplina del alumnado de 4. de Educación Primaria, comparando un grupo control con otro experimental antes y después de una intervención didáctica diferenciada en cada uno de los grupos. 


\section{El pensamiento histórico}

El proceso de enseñanza-aprendizaje de la historia se produce de dos formas principales en las aulas. Por un lado, una educación basada en el uso del libro de texto y en un aprendizaje memorístico de datos, fechas y conceptos. Por otro lado, una enseñanza basada en el constructivismo y metodologías activas que buscan la comprensión de la historia como una narración o interpretación del pasado poniendo énfasis en el método histórico (Santisteban, 2010). La primera forma de enseñanza conlleva la transmisión de la idea de la historia como un conocimiento cerrado y neutral, mientras que la segunda de ellas muestra la historia como un conocimiento discutible y abierto al debate democrático (Miralles y Gómez, 2021; Shemilt, 1987b).

Este segundo enfoque es el que se relaciona con el pensamiento histórico y, por tanto, favorece su desarrollo. Este consiste en que el alumnado no solo memorice hechos, datos y fechas del pasado, denominados conceptos de primer orden, sino que también desarrolle la capacidad de analizar, interpretar y explicar el pasado a partir del análisis crítico de las fuentes, en definitiva, aprender los métodos y técnicas de la investigación histórica que hacen referencia a los conceptos estratégicos de segundo orden (Domínguez, 2015; Seixas y Morton, 2013; Thorp y Persson, 2020; VanSledright, 2004, 2011).

Estos conceptos de segundo orden, atendiendo a Seixas y Morton (2013) serían los siguientes: relevancia histórica, evidencias o fuentes, cambio y continuidad, causas y consecuencias, perspectiva histórica y dimensión ética de la historia. Dichos conceptos, también denominados conceptos metodológicos o habilidades de pensamiento histórico, son los que permiten organizar y dar sentido a los contenidos sustantivos de primer orden y, por tanto, proporcionan el entendimiento de esta disciplina (Lee, 2005; Lee y Ashby, 2000).

Wineburg $(1999,2001)$ señala que es necesaria una enseñanza explicita del pensamiento histórico al no desarrollarse de forma natural, por lo se requiere que, en la enseñanza de la historia, se explique y evidencie cómo se escribe la historia, así como la tarea de los historiadores.

Por ello, para llevar a cabo un adecuado proceso de enseñanza-aprendizaje de la historia es necesario un cambio metodológico y epistemológico (Gómez et al., 2021) en el que el alumnado conozca y comprenda los métodos propios de la disciplina, es decir, cómo se construye el conocimiento histórico. El aprendizaje requiere de una base epistemológica para la comprensión de la historia (Santacana, 2005; Shemilt, 1987b).

Por tanto, para mejorar la educación histórica es preciso un cambio del enfoque de enseñanza que permita la comprensión de qué es la historia y el modo en que esta se construye, es decir, el trabajo de los historiadores, siendo esto la base del pensamiento histórico. Además, se señala la conveniencia de tener en consideración la visión que el alumnado tiene sobre estos aspectos (Valls y López-Facal, 2011).

Sin embargo, se indica como uno de los principales problemas de la educación histórica que esta suele centrarse en la transmisión de los conceptos de primer orden en vez de en la comprensión de la historia como disciplina y el desarrollo de las competencias y habilidades de pensamiento histórico (Lévesque, 2008; Seixas y Morton, 2013; Thorp y Persson, 2020).

En el caso de España, la práctica educativa viene determinada por dos elementos principalmente: el currículo y los libros de texto. Estudios previos han mostrado que la presencia del pensamiento histórico en estos dos elementos es ínfima (Gómez, 2014; Martínez-Hita, 2019; Martínez-Hita y Gómez, 2016, 2018; Sáiz, 2011, 2013; Sáiz y Colomer, 2014). Este hecho supone que el conocimiento del alumnado sobre qué es la historia y el trabajo del historiador, en definitiva, su formación en relación con el pensamiento histórico sea deficiente. 


\section{Concepciones del alumnado sobre la historia}

Las ideas y percepciones del alumnado en torno a la historia y el trabajo de los historiadores ha sido objeto de estudio desde hace décadas. Una de las primeras investigaciones sobre este tema fue desarrollada por Shemilt en los años ochenta del siglo XX (Shemilt, 1987b).

Este autor evaluó los resultados de un proyecto didáctico pionero en la enseñanza de la historia basado en el enfoque del pensamiento histórico. Este proyecto se inició en el año 1972 en Gran Bretaña con el nombre de History Project 13-16 y posteriormente pasó a denominarse School History Project (SHP). Shemilt (1987b) comprobó que el alumnado del SHP, estudiantes de entre trece y dieciséis años, comprendían mejor la naturaleza de la historia que quienes estaban en cursos convencionales. Por ejemplo, los alumnos y alumnas que no habían cursado el SHP definían la historia como "un compendio de hechos preexistentes e inalienables" y "una disciplina memorística", mientras que aquellos estudiantes del proyecto curricular de renovación de la historia expresaban que "Los historiadores elaboran conocimiento a partir de la evidencia, estas elaboraciones son discutibles" y "La Historia es una disciplina de resolución de problemas" (Shemilt, 1987b, p. 188). Shemilt (1987b) defiende, por tanto, que para entender la historia es necesario abordar los aspectos metodológicos y de la explicación histórica, de forma que se comprenda también los métodos, la lógica y perspectivas propias de la disciplina.

Posteriormente, Fuentes (2002) realizó una revisión sobre la visión de la historia por los adolescentes en Estados Unidos y Reino Unido.

En cuanto a Reino Unido, esta autora hace referencia al anterior estudio de Shemilt (1987a) quien estableció cuatro etapas evolutivas en las percepciones del alumnado sobre las fuentes históricas y el trabajo del historiador. Estas ideas evolucionan desde una concepción de la historia como una verdad absoluta que se recoge en los libros de texto hasta la consideración de la historia como una reconstrucción de los eventos del pasado a través del análisis de fuentes. Como se ha indicado anteriormente, fue el alumnado del grupo experimental, es decir, quienes formaron parte del proyecto de innovación en la enseñanza de la historia, quienes desarrollaron una idea de la historia asociada al análisis e interpretación de fuentes frente al grupo control que la consideraban una descripción de hechos del pasado.

Con respecto a las investigaciones llevadas a cabo en Estados Unidos, destacan las realizadas en la década de los 90 por Brophy y VanSledright (1992) y Brophy et al. (1992). La primera investigación (Brophy y VanSledright, 1992) mostró que la mayoría del alumnado consideraba la historia como el estudio de los acontecimientos relevantes del pasado y que tenían dificultades para identificar el trabajo desempeñado por los historiadores. Por su parte, Brophy et al. (1992) completaron el anterior estudio analizando las percepciones del alumnado antes y después de una intervención en el aula para comprobar su evolución. Utilizaron una metodología activa que supuso una mejora en la comprensión de la historia como disciplina y de la tarea del historiador. Por ejemplo, antes de la instrucción, el alumnado desconocía cuál era el papel del historiador y lo confundían con el de un arqueólogo, mientras que después de la intervención didáctica comprendían la tarea del historiador y el análisis de fuentes.

En resumen, tras la revisión de las investigaciones previas, Fuentes (2002) indica que la mayoría de ellas señalan que los discentes consideran la historia como un conocimiento inalterable y cerrado basado en la descripción de los eventos más relevantes del pasado. No obstante, esta visión del alumnado sobre la historia está relacionada con la forma de enseñanza llevada a cabo en el aula, por lo que puede ser modificada mediante el uso de nuevas metodologías. Asimismo, en relación con el trabajo de los historiadores, la mayoría del alumnado considera que se encargan de describir el pasado que ya está escrito en las fuentes o libros, pero no hacen alusión al análisis crítico, interpretación o investigación histórica. Si bien, las explicaciones sobre la tarea de los historiadores de aquellos discentes que han trabajado en clase con fuentes son más 
completas. Por último, alude a la importancia y necesidad de incrementar el número de investigaciones que permitan conocer la visión del alumnado sobre la historia.

Esta misma autora (Fuentes, 2006) realiza años después un análisis de la visión que estudiantes de Educación Secundaria tienen sobre la historia como campo de conocimiento y como materia escolar. Centrándonos en la historia como campo de conocimiento, se le preguntó al alumnado sobre la definición de la historia, su objeto de estudio y el papel del historiador. Los resultados reflejan que la mayoría de los discentes relacionan la historia con el conocimiento del pasado y tienen dificultades para explicar la labor de los historiadores. Sin embargo, aquellos estudiantes que han trabajado con fuentes históricas en el aula tienen una visión más completa tanto del papel del historiador como de la historia como disciplina.

Más actual es la investigación VanSledright y Maggioni (2016) quienes realizan una revisión de los principales estudios llevados a cabo sobre la cognición epistémica en la historia. Estos autores señalan la importancia de entender la epistemología de cada disciplina para mejorar su aprendizaje, ya que, en el caso de la historia, las creencias epistemológicas sobre qué es la historia y cómo se crea su conocimiento están asociadas con el pensamiento histórico y la comprensión histórica. Además, concluyen indicando la necesidad de avanzar en la investigación en esta área con estudios más empíricos y sistemáticos.

Reciente es también la investigación de Fraile-Delgado (2020) que analiza la evolución de las concepciones historiográficas del alumnado de 1. de Educación Secundaria Obligatoria al utilizar una metodología basada en el trabajo de las competencias asociadas al pensamiento histórico.

Antes de la intervención en el aula, en cuanto a la visión de la historia, la mayoría del alumnado la considera como el estudio de los acontecimientos del pasado, seguido por una confusión entre historia y pasado. Respecto a la tarea del historiador, un gran porcentaje desconoce cuál es su labor y lo confunde con el arqueólogo, científico o profesor. Por último, en relación con cómo se estudia el pasado, la mayoría lo desconoce o asocia con la búsqueda de información, si bien un cuarto del alumnado hace alusión al análisis de fuentes.

Tras la intervención didáctica, las concepciones del alumnado han evolucionado favorablemente, especialmente en lo referido al trabajo del historiador, pues gran parte de los discentes identifican al historiador o arqueólogo como quien investiga el pasado mediante el análisis e interpretación de fuentes. Por lo que Fraile-Delgado (2020) concluye que una metodología basada en el pensamiento histórico facilita la evolución positiva de las concepciones historiográficas del alumnado y, por consiguiente, el desarrollo del pensamiento histórico al ser estas fundamentales para la alfabetización histórica de los estudiantes.

Por su parte, Miguel-Revilla et al. (2020) examinan las creencias epistemológicas sobre la historia de futuros docentes tanto de Primaria como Secundaria, es decir, estudiantes universitarios. Combinan un análisis cuantitativo, mediante el instrumento Beliefs About History Questionnaire (BHQ), con un análisis cualitativo, a través de un cuestionario que se centra en cuatro categorías: naturaleza de la historia, interpretación en la historia, objetividad y subjetividad, y el papel de las fuentes. Detectan diferencias entre los futuros docentes de Primaria y los de Secundaria. En cuanto a los de Primaria, aquellos que están en segundo año de carrera confunden el pasado con la historia y no tienen en consideración las fuentes históricas, mientras que los que están en tercer año muestran unas concepciones un poco más completas al haber cursado previamente asignaturas que trataban estos contenidos. Los futuros docentes de Educación Secundaria tienen una visión de la historia como una ciencia y destacan el análisis crítico de las fuentes. Los autores concluyen señalando la importancia de la enseñanza para modificar las creencias epistemológicas. 
Por tanto, estos estudios reflejan un conocimiento inicial erróneo o insuficiente sobre la historia por parte del alumnado, lo cual puede dificultar su comprensión y el desarrollo del pensamiento histórico (Miguel-Revilla et al., 2020; VanSledright y Maggioni, 2016). Además, se señala que el uso de una metodología activa y basada en dicho pensamiento histórico supone modificaciones en las concepciones del alumnado hacia una mejor comprensión del trabajo del historiador y de la historia como disciplina.

Sin embargo, estos estudios previos se han centrado en el análisis del alumnado de Educación Secundaria o Educación Superior (VanSledright y Maggioni, 2016), por lo que existe una laguna de investigación en la etapa de Educación Primaria. Esto es, probablemente, debido a la influencia de la teoría piagetiana que llevó a la consideración de que el alumnado de estas edades difícilmente podía comprender la historia y desarrollar el pensamiento histórico (Éthier et al., 2010; Fuentes, 2002).

No obstante, otras investigaciones han demostrado que el alumnado de Educación Primaria sí es capaz de comprender la historia y pensar históricamente y señalan, además, la importancia del uso de metodologías activas para mejorar la comprensión histórica (Booth, 1983, 1987; Éthier et al., 2010; Fuentes, 2002).

El presente estudio parte de estas ideas y de la laguna de investigación existente en la etapa primaria. Además, pretende completar las investigaciones previas al incluir un análisis cuantitativo de las concepciones del alumnado sobre la historia, de forma que puedan estudiarse las diferencias en la evolución de dichas concepciones estadísticamente. Por ello, su objetivo fue analizar las concepciones del alumnado acerca de la historia como disciplina tras una intervención didáctica, comparando un grupo experimental que trabajó con una metodología activa basada en el pensamiento histórico (Martínez-Hita y Miralles, 2020) con un grupo control que seguía una metodología tradicional basada en el uso del libro de texto.

\section{Método}

\subsection{Objetivos}

El objetivo principal de esta investigación fue conocer cómo evolucionan las concepciones sobre la historia como disciplina del alumnado de 4. de Educación Primaria tras una intervención didáctica en el aula, comparando un grupo experimental que siguió una metodología activa basada en el pensamiento histórico (Martínez-Hita y Miralles, 2020) con un grupo control que trabajó los mismos contenidos mediante una metodología tradicional.

Este objetivo general se desglosó en las siguientes preguntas de investigación:

1. ¿Existen diferencias significativas en las concepciones sobre la historia como disciplina por parte del alumnado del grupo experimental tras la intervención en el aula (pretest-postest)?

- H0: No existen diferencias estadísticamente significativas en el grupo experimental entre el pretest y el postest.

- H1: Existen diferencias estadísticamente significativas en el grupo experimental entre el pretest y el postest.

2. ¿Existen diferencias significativas en las concepciones sobre la historia como disciplina por parte del alumnado del grupo control tras la intervención en el aula (pretest-postest)?

- H0: No existen diferencias estadísticamente significativas en el grupo control entre el pretest y el postest.

- H1: Existen diferencias estadísticamente significativas en el grupo control entre el pretest y el postest. 
3. ¿Existen diferencias significativas en las concepciones sobre la historia como disciplina entre el alumnado del grupo control y experimental?

- H0: No existen diferencias estadísticamente significativas entre el grupo control y experimental en el postest.

- H1: Existen diferencias estadísticamente significativas entre el grupo control y experimental en el postest.

\subsection{Diseño de la investigación}

El presente estudio tuvo un diseño cuasiexperimental con grupo experimental y grupo control, así como con pretest y postest.

\subsection{Participantes}

Los participantes de esta investigación fueron un total de 44 estudiantes de 4.ㅇ de Educación Primaria, esto es, de entre 9 y 10 años de edad, de un colegio público de la Región de Murcia (España).

La selección de la muestra de esta investigación, llevada a cabo durante el curso académico 2017/2018, tuvo un carácter no probabilístico e incidental, ya que se basó en los criterios de accesibilidad a los sujetos, así como de adecuación a los objetivos del estudio (Bisquerra, 2014).

Los participantes estaban divididos en dos grupos que correspondían a sus grupos naturales de clase. Uno de los grupos, formado por 21 estudiantes, fue el grupo control que trabajó con una metodología tradicional, mientras que el grupo experimental estuvo compuesto por 23 discentes y siguió el enfoque basado en el pensamiento histórico. En concreto, en el grupo experimental se implementó un programa de intervención gamificado basado en el pensamiento histórico que incluía metodologías activas e inclusivas como el trabajo cooperativo (Martínez-Hita y Miralles, 2020).

\subsection{Instrumento de recogida de información}

Para la recogida de información se utilizó una prueba de rendimiento elaborada ad hoc con el objetivo de obtener información sobre la visión del alumnado acerca de la historia y la investigación histórica. En concreto, se analizaron los siguientes cuatro ítems de respuesta abierta, así como breve, que formaban parte de dicha prueba (véase Tabla 1).

Tabla 1

Ítems del instrumento de recogida de información y su respectivo concepto

\begin{tabular}{ll}
\hline Ítems & Concepto \\
\hline 1. ¿Qué es para ti la historia? & Definición de la historia \\
2. ¿Cómo se escribe la historia? & Construcción del conocimiento histórico \\
$\begin{array}{l}\text { 3. ¿Quién escribe la historia? } \\
\begin{array}{l}\text { 4. ¿Qué es una fuente histórica? ¿Para qué } \\
\text { sirve? }\end{array}\end{array}$ & Tarea del historiador \\
\hline
\end{tabular}

Esta prueba de rendimiento fue validada utilizando la técnica de juicio de expertos. Los análisis realizados de sus características psicométricas evidenciaron tanto validez de contenido (Escobar-Pérez y Cuervo Martínez, 2008), como una muy alta consistencia interna, siendo el valor de alfa de Cronbach de .850 para estos cuatro ítems (Bisquerra, 1992). 


\subsection{Análisis de la información}

Las respuestas dadas por parte del alumnado fueron codificadas y analizadas con el programa estadístico SPSS v.24. Para llevar a cabo el proceso de codificación y valoración de la información recogida en el pretest y postest se empleó una escala de cinco valores, entre 0 y 4 , asignando a cada valor un determinado indicador de logro para cada uno de los ítems (véase Tabla 2).

Tabla 2

Indicadores de logro para la valoración de cada uno de los ítems

\begin{tabular}{|c|c|c|c|}
\hline ÍTEM & & SCALA E INDICADORES DE LOGRO & EJEMPLO \\
\hline \multirow{5}{*}{$\begin{array}{l}\text { 1. ¿Qué } \\
\text { es para ti } \\
\text { la } \\
\text { historia? }\end{array}$} & 0 & $\begin{array}{l}\text { Desconocimiento. En blanco o } \\
\text { completamente incorrecta. }\end{array}$ & $\begin{array}{l}\text { "Es un libro". "Es un mundo que tiene una } \\
\text { historia" }\end{array}$ \\
\hline & 1 & Nombra algún hecho del pasado. & "Cuando se creó el fuego, la escritura" \\
\hline & 2 & Confusión entre historia y pasado. & $\begin{array}{l}\text { "Es algo que sucedió hace mucho tiempo } \\
\text { como Egipto, la prehistoria" }\end{array}$ \\
\hline & 3 & $\begin{array}{l}\text { Identificación de la historia como } \\
\text { ciencia, pero sin especificar el } \\
\text { objeto de estudio. }\end{array}$ & $\begin{array}{l}\text { "Es la ciencia que investiga". "La historia es } \\
\text { la que investigan los historiadores" }\end{array}$ \\
\hline & 4 & Ciencia que estudia el pasado. & $\begin{array}{l}\text { "Es la ciencia que investiga el pasado de las } \\
\text { personas y sociedades humanas" }\end{array}$ \\
\hline \multirow{5}{*}{$\begin{array}{l}\text { 2. ¿Cómo } \\
\text { se } \\
\text { escribe la } \\
\text { historia? }\end{array}$} & 0 & $\begin{array}{l}\text { Desconocimiento. En blanco o } \\
\text { completamente incorrecta. }\end{array}$ & $\begin{array}{l}\text { "Con letras antiguas". "En dibujos y en } \\
\text { letras" }\end{array}$ \\
\hline & 1 & $\begin{array}{l}\text { No se alude a la investigación. Ya } \\
\text { está escrita. }\end{array}$ & $\begin{array}{l}\text { "Lo buscan por internet". "Creo que se lo } \\
\text { imaginan". "En libros" }\end{array}$ \\
\hline & 2 & Respuesta breve: investigando. & $\begin{array}{l}\text { "Se escribe investigando". "Lo que } \\
\text { investigan, lo escriben". }\end{array}$ \\
\hline & 3 & $\begin{array}{l}\text { Investigando y analizando, sin } \\
\text { nombrar fuentes. O nombra } \\
\text { fuentes pero no habla de } \\
\text { investigar. }\end{array}$ & $\begin{array}{l}\text { "La historia se escribe analizando } \\
\text { información, después la resumen y la } \\
\text { escriben". "Los historiadores tienen que } \\
\text { analizar, investigar y con esa información } \\
\text { van escribiendo la historia" }\end{array}$ \\
\hline & 4 & $\begin{array}{l}\text { Se hace alusión a la investigación } \\
\text { y al análisis de fuentes. }\end{array}$ & $\begin{array}{l}\text { "Analizando fuentes históricas e } \\
\text { investigándolas". "Buscaban las fuentes } \\
\text { históricas y analizaban y la escriben" }\end{array}$ \\
\hline \multirow{5}{*}{$\begin{array}{l}\text { 3. ¿Quién } \\
\text { escribe la } \\
\text { historia? }\end{array}$} & 0 & $\begin{array}{l}\text { Desconocimiento. En blanco o } \\
\text { completamente incorrecta. }\end{array}$ & "Dios" \\
\hline & 1 & Las personas. & "Las personas que lo han estudiado" \\
\hline & 2 & Escritor, autor libro. & $\begin{array}{l}\text { "Un escritor e ilustrador". "El autor del } \\
\text { libro" }\end{array}$ \\
\hline & 3 & $\begin{array}{l}\text { Científico, } \\
\text { investigador. }\end{array}$ & "Los arqueólogos". "Los investigadores" \\
\hline & 4 & Historiadores. & "Los historiadores e historiadoras" \\
\hline \multirow{3}{*}{$\begin{array}{l}\text { 4. ¿Qué } \\
\text { es una } \\
\text { fuente } \\
\text { histórica } \\
\text { ? ¿Para } \\
\text { qué } \\
\text { sirve? }\end{array}$} & 0 & $\begin{array}{l}\text { Desconocimiento. En blanco o } \\
\text { completamente incorrecta }\end{array}$ & "Sirve para decir si es escrita, oral" \\
\hline & 1 & $\begin{array}{l}\text { Solo indica que da información sin } \\
\text { especificar del pasado. }\end{array}$ & $\begin{array}{l}\text { "Una fuente es lo que te da información y } \\
\text { sirve para acumular información" }\end{array}$ \\
\hline & 2 & $\begin{array}{l}\text { Ejemplo de fuente sin explicar } \\
\text { para qué sirve. } \\
\text { Dice para qué sirve, pero no qué } \\
\text { es. }\end{array}$ & $\begin{array}{l}\text { "Es un texto que habla sobre la historia". } \\
\text { "Es un sitio donde hay muchas cosas como } \\
\text { unas ruinas, cuevas pintadas" }\end{array}$ \\
\hline
\end{tabular}


3 Explicación correcta a falta de "Es un objeto del pasado que sirve para ver decir que con su análisis se escribe cómo las personas vivían antes" la historia

4 Explicación correcta y en detalle. "Las huellas del pasado. Sirve para analizarlos y después escribir la historia"

En el análisis estadístico se calcularon los descriptivos, las frecuencias y las diferencias intergrupos e intragrupo.

La comparación intragrupal se realizó a través del no paramétrico de $\mathrm{W}$ de Wilcoxon y la comparación entre el grupo control y experimental se llevó a cabo con el estadístico U de MannWhitney. La significación estadística se estableció con un valor de $p \leq .05$.

Mediante la $r$ de Rosenthal (Rosenthal, 1991), se calculó también el tamaño del efecto (TE) para medir la magnitud de las diferencias en las comparaciones intergrupos e intragrupo.

\section{Resultados}

Los resultados se presentan atendiendo a las preguntas de investigación.

1. ¿Existen diferencias significativas en las concepciones sobre la historia como disciplina por parte del alumnado del grupo experimental tras la intervención en el aula (pretest-postest)?

La Tabla 3 muestra los estadísticos descriptivos del grupo experimental en el pretest. Se observaron puntuaciones muy bajas en los cuatro ítems, siendo la moda el valor de cero en tres de ellos, es decir, la respuesta más repetida que equivalía con un desconocimiento sobre los aspectos preguntados.

Tabla 3

Estadísticos descriptivos del grupo experimental en el pretest

\begin{tabular}{lrrrrrrr}
\hline & N & Media & Mediana & Moda & $\begin{array}{c}\text { Desviación } \\
\text { estándar }\end{array}$ & Mínimo & Máximo \\
& & & & & & & \\
\hline Item_1 & 23 & 1.13 & 2.00 & 2 & .968 & 0 & 2 \\
Item_2 & 23 & .96 & 1.00 & 0 & .928 & 0 & 3 \\
Item_3 & 23 & .83 & 1.00 & 0 & .887 & 0 & 3 \\
Item_4 & 23 & .39 & .00 & 0 & .891 & 0 & 3 \\
\hline
\end{tabular}

Los estadísticos descriptivos del postest del grupo experimental se pueden observar en la Tabla 4. Se produjo un aumento en las puntuaciones de todos los ítems que reflejaron una mejora en los conocimientos sobre la historia por parte del alumnado tras la intervención en el aula. Tanto la mediana como la media de los cuatro ítems fue igual o superior a dos, esto es, el valor medio de la escala de valoración de las respuestas.

Tabla 4

Estadísticos descriptivos del grupo experimental en el postest

\begin{tabular}{llrrrrrr}
\hline & N & Media & Mediana & Moda & $\begin{array}{c}\text { Desviación } \\
\text { estándar }\end{array}$ & Mínimo & Máximo \\
& & & & & & & \\
\hline Item_1 & 23 & 2.83 & 3.00 & 4 & 1.370 & 0 & 4 \\
Item_2 & 23 & 2.87 & 3.00 & 3 & 1.180 & 0 & 4 \\
Item_3 & 23 & 3.91 & 4.00 & 4 & .288 & 3 & 4 \\
Item_4 & 23 & 2.00 & 2.00 & 0 & 1.567 & 0 & 4 \\
\hline
\end{tabular}


Para determinar si este aumento en las puntuaciones en el postest respecto al pretest es significativo, así como para dar respuesta a la primera pregunta de la investigación, se realizó la prueba de contraste no paramétrica para dos muestras dependientes o relacionadas $\mathrm{W}$ de Wilcoxon (véase Tabla 5). Los resultados permitieron rechazar la hipótesis nula y aceptar la alterna, es decir, existieron diferencias estadísticamente significativas en todos los ítems en el grupo experimental entre el pretest y el postest $(p<.05)$.

Además, se calculó el tamaño del efecto (TE), esto es, cuán grandes fueron estas diferencias, e indicó unas modificaciones moderadas en las concepciones sobre la historia como disciplina del alumnado del grupo experimental, atendiendo a los criterios de Cohen (1988).

Tabla 5

Pruebas $W$ de Wilcoxon ${ }^{\text {a }}$ del postest/pretest del grupo experimental con su respectivo tamaño del efecto

\begin{tabular}{|c|c|c|c|}
\hline Ítem & $\begin{array}{c}\text { Med } \\
\text { ia }\end{array}$ & $\begin{array}{c}Z \\
\text { (Sig.) }\end{array}$ & TE \\
\hline Item_1_pre & 1.13 & \multirow{2}{*}{$\begin{array}{c}-3.859^{b} \\
(.000)\end{array}$} & \multirow[t]{2}{*}{0.569} \\
\hline Item_1_pos & 2.83 & & \\
\hline Item_2_pre & .96 & \multirow{2}{*}{$\begin{array}{c}-3.966^{b} \\
(.000)\end{array}$} & \multirow[t]{2}{*}{0.585} \\
\hline Item_2_pos & 2.87 & & \\
\hline Item_3_pre & .83 & \multirow{2}{*}{$\begin{array}{c}-4.255^{b} \\
(.000)\end{array}$} & \multirow[t]{2}{*}{0.627} \\
\hline Item_3_pos & 3.91 & & \\
\hline Item_4_pre & .39 & \multirow{2}{*}{$\begin{array}{c}-3.548^{b} \\
(.000)\end{array}$} & \multirow[t]{2}{*}{0.523} \\
\hline Item_4_pos & 2.00 & & \\
\hline
\end{tabular}

a. Prueba de rangos con signo de Wilcoxon

b. Se basa en rangos negativos.

Desde un punto de vista más cualitativo, en cuanto al primer ítem sobre qué es la historia, la mayoría del alumnado en el pretest confundía el pasado con la historia (52.2\%) o desconocía qué era (39.1\%). En el postest, un alto porcentaje de los discentes (43.5\%) definían la historia como una ciencia que estudia el pasado, aunque seguía existiendo un $21.7 \%$ que confundió la historia con el pasado. Un ejemplo de la evolución en las respuestas se constató en uno de los discentes que en el pretest definió la historia como "Cosas que han pasado hace mucho tiempo" y en el postest indicó que "Para mí la historia es la ciencia que investiga el pasado" (discente 8).

Respecto al segundo ítem relativo a cómo se construye el conocimiento histórico, en el pretest, la mayoría del alumnado (39.1\%) lo desconocía, seguido por un 30.4\% que indicaban que la historia aparece en los libros e internet sin aludir a la investigación. El resto señalaban la investigación pero de manera escueta y sin nombrar las fuentes históricas, por ejemplo, "Se escribe investigando" (discente 2). En el postest se observó una evolución en las respuestas. Un $30.4 \%$ hacían referencia a la investigación y el análisis de fuentes, como "Buscaban las fuentes históricas y analizaban y la escriben" (discente 15) y un $47.8 \%$ indicaban el proceso de investigación y análisis pero sin nombrar las fuentes o nombraban las fuentes pero no la investigación, "Hay que investigar, analizarlo y cuando han terminado lo escriben" (discente 7).

En relación con el tercer ítem, ¿quién escribe la historia?, la mayoría del alumnado (43.5\%) lo desconocía en el pretest, indicaban que eran las personas (34.8\%) o que eran los autores y escritores de libros (17.4\%). En el postest, estas respuestas se vieron modificadas al indicar el 91.3\% que eran los historiadores e historiadoras. Esto concuerda con los resultados obtenidos cuantitativamente respecto al tamaño del efecto ya que fue este ítem el que obtuvo el mayor tamaño del efecto (0.627) (véase Tabla 5). 
Por último, en cuanto a las fuentes históricas, la gran mayoría del alumnado en el pretest (82.6\%) desconocía qué eran y para qué servían. Mientras que en el postest, sus respuestas fueron más variadas. Un $21.7 \%$ ofrecieron una respuesta correcta y detallada, como el discente 13 que indicó "Es algo que se conserva del pasado y sirve para escribir la historia". Sin embargo, continuó existiendo un $30.4 \%$ de los discentes que no fueron capaces de explicar su significado y utilidad. Sirva de ejemplo de evolución un alumno que desconocía las fuentes históricas en el pretest, ya que dejó su respuesta en blanco, y pasó a definirlas como "huellas del pasado que sirven para escribir la historia" (discente 2).

\section{2. ¿Existen diferencias significativas en las concepciones sobre la historia como disciplina por parte del alumnado del grupo control tras la intervención en el aula (pretest-postest)?}

Los estadísticos descriptivos del grupo control en el pretest se observan en la Tabla 6. Todos los ítems obtuvieron puntuaciones muy bajas que evidenciaron unos conocimientos insuficientes sobre la historia y la investigación histórica.

Tabla 6

Estadísticos descriptivos del grupo control en el pretest

\begin{tabular}{lrrrrrrr}
\hline & N & Media & Mediana & Moda & $\begin{array}{c}\text { Desviación } \\
\text { estándar }\end{array}$ & Mínimo & Máximo \\
& & & & & & & \\
\hline Item_1 & 21 & 1.19 & 2.00 & 2 & .928 & 0 & 2 \\
Item_2 & 21 & .90 & .00 & 0 & 1.044 & 0 & 3 \\
Item_3 & 21 & 1.14 & .00 & 0 & 1.352 & 0 & 3 \\
Item_4 & 21 & .24 & .00 & 0 & .889 & 0 & 4 \\
\hline
\end{tabular}

Los descriptivos del postest del grupo control mostraron un aumento en las puntuaciones de los ítems (véase Tabla 7). No obstante, y a excepción del ítem 3, estas puntuaciones continúan siendo bajas, especialmente en el caso del ítem número 4.

Tabla 7

Estadísticos descriptivos del grupo control en el postest

\begin{tabular}{lrrrrrrr}
\hline & N & Media & Mediana & Moda & $\begin{array}{c}\text { Desviación } \\
\text { estándar }\end{array}$ & Mínimo & Máximo \\
& & & & & & & \\
\hline Item_1 & 21 & 1.29 & 2.00 & 2 & 1.007 & 0 & 3 \\
Item_2 & 21 & 1.33 & 2.00 & 0 & 1.197 & 0 & 3 \\
Item_3 & 21 & 2.48 & 3.00 & 4 & 1.750 & 0 & 4 \\
Item_4 & 21 & .86 & .00 & 0 & 1.315 & 0 & 4 \\
\hline
\end{tabular}

Para dar respuesta a la segunda pregunta de investigación, ¿̇existen diferencias significativas en las concepciones sobre la historia como disciplina por parte del alumnado del grupo control tras la intervención en el aula (pretest-postest)? se realizó la prueba W de Wilcoxon. Como muestra la Tabla 8, no existieron diferencias significativas entre el pretest y el postest en los ítems 1 y 2 , pero sí en el caso de los ítems 3 y $4(p<.05)$. El tamaño del efecto (TE) fue pequeño, es decir, las diferencias fueron pequeñas al situarse por debajo del valor de .50 (Cohen, 1988).

Por tanto, se rechazó la hipótesis nula y aceptó la alterna en el caso de los ítems 3 y 4 , esto es, existieron diferencias estadísticamente significativas en estos ítems en el grupo control entre el pretest y el postest $(p<.05)$, pero se aceptó la hipótesis nula en el caso de los ítems 1 y 2. 
Tabla 8

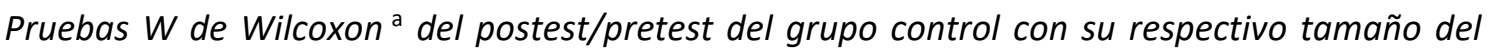
efecto

\begin{tabular}{|c|c|c|c|}
\hline \multirow[b]{2}{*}{ Ítem } & \multicolumn{3}{|c|}{$Z$} \\
\hline & Media & (Sig.) & TE \\
\hline Item_1_pre & 1.19 & $-.649^{b}$ & .100 \\
\hline Item 1 pos & 1.29 & (.516) & \\
\hline Item_2_pre & .90 & $-1.852^{b}$ & .286 \\
\hline Item_2_pos & 1.33 & $(.064)$ & \\
\hline Item_3_pre & 1.14 & $-2.954^{b}$ & .456 \\
\hline Item_3_pos & 2.48 & (.003) & \\
\hline Item_4_pre & .24 & $-1.994^{b}$ & .308 \\
\hline Item_4_pos & .86 & $(.046)$ & \\
\hline
\end{tabular}

a. Prueba de rangos con signo de Wilcoxon

b. Se basa en rangos negativos.

En una aproximación más cualitativa a las respuestas del alumnado al primer ítem relativo a qué es la historia, en el pretest más de la mitad de los discentes (52.4\%) confundían la historia con el pasado y un 33.3\% desconocía qué era. En el postest se mantuvieron unos porcentajes prácticamente iguales al pretest que se refleja en la ausencia de diferencias estadísticamente significativas en el análisis cuantitativo. Por ejemplo, la respuesta de un discente a este ítem en el pretest fue "Para mí es lo que ha ocurrido en el pasado" y en el postest "Para mí son los hechos que han ocurrido en el pasado" (discente 35).

En cuanto al segundo ítem sobre cómo se escribe la historia, en el pretest, un 52.4\% de los discentes lo desconocían, seguido por un 33.3\% que solamente nombran la investigación. En el postest, se reduce el número de estudiantes que no conocen cómo se construye el conocimiento histórico $(38.1 \%)$, se mantiene un $33.3 \%$ que hace referencia de manera sucinta a la investigación y se incrementa a un $19 \%$ el alumnado que aporta una mejor explicación sobre la investigación, aunque todavía incompleta. Por ejemplo, se dieron respuestas en el postest como "Investigando cosas del pasado" (discente 34), "Investigando por todos los lugares" (discente 35) o "Con las fuentes históricas encontradas" (discente 41).

En relación con el tercer ítem sobre quién escribe la historia, aproximadamente la mitad del alumnado (52.4\%) lo desconocía, pero un $28.6 \%$ nombraron a los arqueólogos o investigadores. En cuanto al postest, el porcentaje de estudiantes que no sabían quienes escribían la historia se redujo a un $28.6 \%$, además, un $23.8 \%$ señalaba a los arqueólogos o investigadores y un $42.9 \%$ nombró a los historiadores.

Finalmente, en relación con las fuentes históricas, al igual que en el grupo experimental, la mayoría desconocía en el pretest qué eran y su utilidad (90.5\%). En el postest, aunque se redujo este porcentaje, continuó existiendo un $66.7 \%$ que no sabían dar respuesta a esta pregunta, seguido por un $19 \%$ que indicaron algún ejemplo de fuente histórica o explicaron su utilidad, pero no su significado. Por ejemplo, "Los mapas" (discente 26) o "Son objetos del pasado" (discente 41).

\section{3. ¿Existen diferencias significativas en las concepciones sobre la historia como disciplina entre el alumnado del grupo control y experimental?}

Antes de responder a la tercera pregunta de investigación, se comprobó que no existían diferencias previas entre los dos grupos antes de la intervención didáctica en cada uno de ellos. 
La Tabla 9 muestra la comparación de los resultados obtenidos en el pretest por el grupo control y experimental. La prueba de contraste no paramétrica para dos muestras independientes $U$ de Mann-Whitney evidenció que no existían diferencias estadísticamente significativas entre el grupo control y experimental en ninguno de los ítems $(p>.05)$.

Tabla 9

Prueba $U$ de Mann-Whitney del pretest entre grupo control y grupo experimental

\begin{tabular}{|c|c|c|c|c|c|}
\hline & Grupo & $\begin{array}{c}\text { Rango } \\
\text { promedi } \\
0\end{array}$ & $\begin{array}{c}\text { Suma de } \\
\text { rangos }\end{array}$ & $\begin{array}{c}U \\
\text { (Sig.) }\end{array}$ & $Z$ \\
\hline Item & Experimental & 22.20 & 510.50 & \multirow{2}{*}{$\begin{array}{c}234.500 \\
(.855)\end{array}$} & \multirow{2}{*}{-.183} \\
\hline$\_1$ & Control & 22.83 & 479.50 & & \\
\hline Item & Experimental & 23.00 & 529.00 & \multirow{2}{*}{$\begin{array}{c}230.000 \\
(.772)\end{array}$} & \multirow{2}{*}{-.289} \\
\hline 2 & Control & 21.95 & 461.00 & & \\
\hline Item & Experimental & 21.78 & 501.00 & \multirow{2}{*}{$\begin{array}{c}225.000 \\
(.678)\end{array}$} & \multirow{2}{*}{-.415} \\
\hline 3 & Control & 23.29 & 489.00 & & \\
\hline Item & Experimental & 23.33 & 536.50 & \multirow{2}{*}{$\begin{array}{c}222.500 \\
(.454)\end{array}$} & \multirow{2}{*}{-.749} \\
\hline-4 & Control & 21.60 & 453.50 & & \\
\hline
\end{tabular}

Tras comprobar la ausencia de diferencias previas entre los grupos, se llevó a cabo la comparación de los resultados del postest para poder dar respuesta a la tercera pregunta de investigación, esto es, ¿existen diferencias significativas en las concepciones sobre la historia como disciplina entre el alumnado del grupo control y experimental?

La prueba $U$ de Mann-Whitney mostró que existían diferencias en todos los ítems entre el grupo control y experimental, siendo los resultados obtenidos por el grupo experimental mayores a los del control. Por lo que se rechazó la hipótesis nula y se aceptó la alterna, es decir, existieron diferencias estadísticamente significativas entre los rangos promedios de todos los ítems del postest (véase Tabla 10). Además, estas diferencias, a excepción del ítem 4, fueron moderadas como reflejaron los valores del tamaño del efecto (TE), superiores a .50 (Cohen, 1988).

Tabla 10

Prueba $U$ de Mann-Whitney del postest entre grupo control y grupo experimental con su respectivo tamaño del efecto

\begin{tabular}{|c|c|c|c|c|c|c|}
\hline & Grupo & $\begin{array}{c}\text { Rango } \\
\text { promedi } \\
0 \\
\end{array}$ & $\begin{array}{c}\text { Suma de } \\
\text { rangos }\end{array}$ & $\begin{array}{c}U \\
\text { (Sig.) }\end{array}$ & $Z$ & $\mathrm{TE}$ \\
\hline \multirow[t]{2}{*}{ Item_1 } & Experimental & 29.20 & 671.50 & \multirow{2}{*}{$\begin{array}{l}87.500 \\
(.000)\end{array}$} & \multirow{2}{*}{-3.760} & \multirow{2}{*}{.567} \\
\hline & Control & 15.17 & 318.50 & & & \\
\hline \multirow[t]{2}{*}{ Item_2 } & Experimental & 29.39 & 676.00 & \multirow{2}{*}{$\begin{array}{l}83.000 \\
(.000)\end{array}$} & \multirow{2}{*}{-3.849} & \multirow{2}{*}{.580} \\
\hline & Control & 14.95 & 314.00 & & & \\
\hline \multirow[t]{2}{*}{ Item_3 } & Experimental & 27.89 & 641.50 & \multirow{2}{*}{$\begin{array}{c}117.500 \\
(.000)\end{array}$} & \multirow{2}{*}{-3.542} & \multirow{2}{*}{.534} \\
\hline & Control & 16.60 & 348.50 & & & \\
\hline \multirow[t]{2}{*}{ Item_4 } & Experimental & 26.80 & 616.50 & \multirow{2}{*}{$\begin{array}{c}142.500 \\
(.013)\end{array}$} & \multirow{2}{*}{-2.485} & \multirow{2}{*}{.375} \\
\hline & Control & 17.79 & 373.50 & & & \\
\hline
\end{tabular}




\section{Discusión y conclusiones}

A pesar del progresivo protagonismo del pensamiento histórico y la defensa de una innovación didáctica desde las investigaciones sobre la educación histórica (Domínguez, 2015; Lévesque y Clark, 2018; Seixas y Morton, 2013; Thorp y Persson, 2020), los resultados obtenidos, que mostraron una visión inicial sobre la historia deficiente antes de la intervención didáctica en ambos grupos, coinciden con las investigaciones llevadas a cabo hace ya varias décadas (Brophy y VanSledright, 1992; Brophy et al., 1992; Fuentes, 2002, 2006).

No obstante, tras la intervención en el aula, se evidenció una evolución en las concepciones de ambos grupos. En el grupo experimental esta evolución fue mayor, existiendo diferencias significativas en todos los ítems y un tamaño del efecto moderado. Además, todos los ítems superaron, tanto en su media como mediana, el valor medio de la escala de valoración de las respuestas, lo que supone un conocimiento más que suficiente. Por su parte, aunque el grupo control también mejoró sus concepciones, estas modificaciones fueron menores. Las diferencias fueron significativas en solo dos de los cuatro ítems y el tamaño del efecto pequeño. A esto hay que añadir que solo la media de uno de los ítems obtuvo una puntuación superior al valor medio de la escala.

Asimismo, la comparación intergrupal reflejó la existencia de diferencias estadísticamente significativas entre el grupo experimental y control en el postest, las cuales no existían en el pretest. Por tanto, estos resultados indican que el tipo de metodología empleada ha influido en las modificaciones de las concepciones del alumnado sobre la historia como disciplina. El alumnado del grupo experimental, que seguía un enfoque de enseñanza basado en el pensamiento histórico y en metodologías activas, tuvo una visión más completa de la historia que aquellos del grupo control que trabajaron con una metodología tradicional basada en el uso del libro de texto.

Estos resultados concuerdan con las investigaciones previas (Brophy et al., 1992; Fraile-Delgado, 2020; Fuentes, 2002, 2006; Shemilt, 1987a, 1987b) que señalaban la mejor comprensión de la historia como disciplina y del trabajo de los historiadores por parte de aquellos discentes que trabajaron en el aula con una metodología activa y basada en el pensamiento histórico. Por consiguiente, se coincide con la importancia y trascendencia de la metodología como un factor relevante en las modificaciones de las concepciones del alumnado sobre la investigación histórica.

Además, como señalan VanSledright y Maggioni (2016), si se persigue la mejora en el aprendizaje de la historia es necesario una comprensión de la epistemología específica de la disciplina, ya que la visión sobre qué es la historia y cómo se crea su conocimiento está relacionado con el pensamiento histórico y, por ende, con la comprensión histórica.

El uso de una metodología activa basada en el pensamiento histórico permite trabajar en el aula los aspectos metodológicos de la disciplina y, de esta forma, conocer cómo se construye el conocimiento histórico. Esta base epistemológica se hace necesaria para una adecuada enseñanza y comprensión de la historia, pues se consigue avanzar desde una concepción de la historia como un conocimiento neutral y cerrado hacia la consideración de la historia como una construcción, una narración o interpretación del pasado a partir de evidencias (Santisteban, 2010; Shemilt, 1987b).

Consecuentemente, es posible dar solución a una de las principales problemáticas de la educación histórica que es señalada desde la investigación en este campo de estudio, esto es, el protagonismo de los conceptos sustantivos de primer orden frente a la comprensión de la historia (Lévesque, 2008; Martínez-Hita y Gómez, 2018). Se consigue así mejorar la educación histórica al lograr tanto un cambio metodológico como epistemológico (Gómez et al., 2021). 
Asimismo, se constata que sería factible desarrollar un conocimiento de la historia con alumnado de Educación Primaria, como se señalaba en otras investigaciones previas (Booth, 1983, 1987; Éthier et al., 2010; Fuentes, 2002), frente a las ideas difundidas, debido a la influencia de la teoría piagetiana, sobre la imposibilidad de comprender la historia por discentes de estas edades (Éthier et al., 2010; Fuentes, 2002).

Esta investigación ha mostrado la capacidad del alumnado de 4. de Educación Primaria para evolucionar desde unas concepciones erróneos o deficientes sobre la historia como disciplina hacia una visión más completa de la misma, especialmente si se trabaja mediante una metodología activa basada en el pensamiento histórico. Por este motivo, se propone un aprendizaje por parte del alumnado, ya desde edades tempranas, sobre cómo se construye el conocimiento histórico y la tarea de los historiadores, como ya se sugiere desde la investigación en didáctica de la historia (VanSledright, 2011). Este aprendizaje sería conveniente realizarlo mediante metodologías que permitan superar el papel protagonista de los libros de texto y la clase magistral y avanzar hacia un enfoque basado en el pensamiento histórico combinado con metodologías activas, estando así en consonancia con las actuales tendencias educativas (Gómez et al., 2018; Muntaner et al., 2020).

Finalmente, señalar que se debe tener en consideración las limitaciones propias de esta investigación como es el tamaño muestral y la existencia de un único investigador en la valoración de las respuestas del alumnado. Por ello, sería recomendable para futuras investigaciones sobre esta temática ampliar el número de participantes y los niveles educativos analizados, así como incluir más investigadores permitiendo una triangulación de los datos y así eliminar el posible sesgo de un único investigador.

\section{Referencias bibliográficas}

Bisquerra, R. (coord.) (2014). Metodología de la investigación educativa (4a ed.). Madrid: La Muralla.

Bisquerra, R. (1992). Introducción a la estadística aplicada a la investigación educativa. Madrid: PPU.

Booth, M.B. (1983). Skills, concepts and attitudes: the development of adolescent children's historical thinking. History and theory, 22, 101-107.

Booth, M. B. (1987). Ages and concepts: a critique of the piagetian approach to history teaching. En C. Portal (Ed.), The history curriculum for teachers (pp. 22-38). Londres: The Falmer Press.

Brophy, J. y VanSledright, B. (1992). Storytelling, imagination and fanciful elaboration in a children's historical reconstructions. American Educational Research Journal, 20(4), 837859.

Brophy, J., VanSledright, B. y Brendin, S. (1992). Fifh grader's ideas about history expressed before and after the instruction to the subject. Theory and research in social education, $X X(4), 440-489$.

Cohen, J. (1988). Statistical Power Analysis for the Behavioral Sciences (2nd ed.). New Jersey: Lawrence Erlbaum Associates.

Domínguez, J. (2015). Pensamiento histórico y evaluación de competencias. Barcelona: Graó.

Escobar-Pérez, J. y Cuervo-Martínez, Á. (2008). Validez de contenido y juicio de expertos: una aproximación a su utilización. Avances en Medición, 6, 27-36. 
Éthier, M. A., Demers, S. y Lefrançois, D. (2010). Las investigaciones en didáctica sobre el desarrollo del pensamiento histórico en la enseñanza primaria. Una panorámica de la literatura publicada en francés e inglés desde el año 1990. Enseñanza de las ciencias sociales, 9, 61-74.

Fraile-Delgado, F. J. (2020). Pensar en Clío: la evolución de las concepciones historiográficas del alumnado de 10 de Educación Secundaria Obligatoria. Didáctica de las ciencias experimentales y sociales, 39, 3-16. https://doi.org/10.7203/DCES.39.15512

Fuentes, C. (2002). La visión de la historia por los adolescentes: revisión del estado de la cuestión en Estados Unidos y el Reino Unido. Enseñanza de las ciencias sociales, 1, 55-68.

Fuentes, C. (2006). Concepciones de los alumnos sobre la Historia. Educar em Revista, 22, 219239.

Gómez, C. J. (2014). Pensamiento histórico y contenidos disciplinares en los libros de texto. Un análisis exploratorio de la Edad Moderna en 2.. de la ESO. Ensayos. Revista de la Facultad de Educación de Albacete, 29(1), 131-158.

Gómez, C. J., Rodríguez-Medina J., Miralles, P., Arias, V.B. (2021). Efectos de un programa de formación del profesorado en la motivación y satisfacción de los estudiantes de historia en enseñanza secundaria. Revista de Psicodidáctica, 26, 45-52. https://doi.org/10.1016/j.psicoe.2020.08.001

Gómez, C.J., Ortuño, J. y Miralles, P. (2018). Enseñar ciencias sociales con métodos activos de aprendizaje. Reflexiones y propuestas a través de la indagación. Octaedro.

Lee, P. (2005). Putting Principles into Practice: Understanding History. En M. S. Donovan y J. D. Bransford (Eds.), How students learn: History in the classroom (pp. 29-78). Washington, DC: National Academies Press.

Lee, P. y Ashby, R. (2000). Progression in Historical Understanding among Students Ages 7-14. En P. N. Stearns, P. Seixas y Wineburg, S. (Eds.), Knowing, Teaching, and Learning History: National and International Perspectives (pp. 199-222). New York, NY: New York University Press.

Lévesque, S. (2008). Thinking historically: Educating students for the twenty-first century. Toronto: Buffalo.

Lévesque, S., y Clark, P. (2018). Historical Thinking: Definitions and Educational Applications. En S. Metzger y L. M. Harris (Eds.), The Wiley International Handbook of History Teaching and Learning (pp. 119-148). Hoboken, NJ: John Wiley \& Sons, Inc. https://doi.org/10.1002/9781119100812.ch5

Martínez-Hita, M (2019). Análisis comparativo del modelo cognitivo y conceptos históricos en el currículum español e inglés. En EIDUM (coord.), IV Jornadas Doctorales Escuela Internacional de Doctorado de la Universidad de Murcia (EIDUM) (pp. 560-563). Murcia: Editum.

Martínez-Hita, M. y Gómez, C. J. (2016). Diferentes enfoques sobre la enseñanza de la historia y del pensamiento histórico en el currículo español y canadiense. En J. Maquilón, C. J. Gómez y M. B. Alfageme (Eds.), De la investigación a la mejora educativa en las aulas (pp. 19-32). Murcia: Editum.

Martínez-Hita, M. y Gómez, C. J. (2018). Nivel cognitivo y competencias de pensamiento histórico en los libros de texto de Historia de España e Inglaterra. Un estudio comparativo. Revista de Educación, 379, 145-169. https://doi.org/10.4438/1988-592X-RE-2017-379$\underline{364}$ 
Martínez-Hita, M. y Miralles, P. (2020). Evaluación del diseño de un programa de intervención gamificado para el desarrollo del pensamiento histórico en Educación Primaria. RIFOP, 34(2), 187-204. https://doi.org/10.47553/rifop.v34i2.78420

Miguel-Revilla, D., Carril-Merino, T. y Sánchez-Agustí, M. (2020). An examination of epistemic beliefs about history in initial teacher training: a comparative analysis between primary and secondary education prospective teachers. The Journal of Experimental Education, 89, 54-73. https://doi.org/10.1080/00220973.2020.1718059

Miralles, P. y Gómez, C. J. (2021). El pensamiento histórico del alumnado y su relación con las competencias. En Educación histórica para el siglo XXI. Principios epistemológicos y metodológicos. Cali: Universidad del Valle y Universidad Icesi.

Muntaner, J. J., Pinya, C. y Mut, B. (2020). El impacto de las metodologías activas en los resultados académicos. Profesorado. Revista de Currículum y Formación de Profesorado, 24(1), 96-114. https://doi.org/10.30827/profesorado.v24i1.8846

Rosenthal, R. (1991). Meta- analytic procedures for social research (2nd ed.). Newbury Park, CA: Sage.

Sáiz, J. (2011). Actividades de libros de texto de Historia, competencias básicas y destrezas cognitivas, una difícil relación: análisis de manuales de 1ㅇ y 2ㅇ de ESO. Didáctica de las ciencias experimentales y sociales, 25, 37-64.

Sáiz, J. (2013). Alfabetización histórica y competencias básicas en libros de texto de historia y en aprendizajes de estudiantes. Didáctica de las ciencias experimentales y sociales, 27, 4366. https://doi.org/10.7203/dces.27.2648

Sáiz, J. y Colomer, J. C. (2014). ¿Se enseña pensamiento histórico en libros de texto de Educación Primaria? Análisis de actividades de historia para alumnos de 10-12 años de edad. CLIO. History and History teaching, 40.

Santacana, J. (2005). Reflexiones en torno al laboratorio escolar de ciencias sociales, íber: Didáctica de las Ciencias Sociales, Geografía e Historia, 43, 7-14.

Santisteban, A. (2010). La formación de competencias de pensamiento histórico. Clío \& Asociados, 14), 34-56.

Seixas, P. y Morton, T. (2013). The Big Six Historical Thinking Concepts. Toronto: Nelson Education Ltd.

Shemilt, D. (1987a). Adolescent ideas about evidence and methodology in History. En C. Portal (Ed.), The history curriculum for teachers (pp. 39-61). Londres: The Falmer Press.

Shemilt, D. J. (1987b). El proyecto «Historia 13-16» del Schools Council: pasado, presente y futuro. En M.E.C., La geografía y la Historia dentro de las Ciencias Sociales: hacia un currículum integrado (pp. 173-208). Madrid: Centro de Publicaciones del M.E.C.

Thorp, R. y Persson, A. (2020). On historical thinking and the history educational challenge. Educational Philosophy and Theory, 52(8), 891-901, https://doi.org/10.1080/00131857.2020.1712550

Valls, R., y López-Facal, R. (2011). ¿ Un nuevo paradigma para la enseñanza de la historia? Los problemas reales y las polémicas interesadas al respecto en España y en el contexto del mundo occidental. Enseñanza de las ciencias sociales, 10, 75-86.

VanSledright, B. A. (2004). What Does It Mean to Think Historically... and How Do You Teach It? Social Education, 68(3), 230-233. 
VanSledright, B. A. (2011). The Challenge of Rethinking History Education. On Practice, Theories, and Policy. Nueva York: Routledge

VanSledright, B. A., y Maggioni, L. (2016). Epistemic cognition in history. In J. A. Greene, W. A. Sandoval, y I. Bråten (Eds.), Handbook of epistemic cognition (pp. 128-146). New York, NY: Routledge.

Wineburg, S. (1999). Historical Thinking and Other Unnatural Acts. The Phi Delta Kappan, 80(7), 488-499.

Wineburg, S. (2001). Historical Thinking and other Unnatural Acts. Charting the Future of Teaching the Past. Filadelfia: Temple University Press. 